\title{
Immunosuppression: practice and trends
}

\author{
Dixon B. Kaufman ${ }^{a, *}$, Ron Shapiro ${ }^{b}$, Michael R. \\ Lucey $^{c}$, Wida S. Cherikh ${ }^{\text {, }}$ Rami T. Bustamie \\ and David B. Dykef \\ a Northwestern University, Feinberg School of Medicine, \\ Chicago, IL; \\ b University of Pittsburgh, Pittsburgh, PA; \\ 'University of Wisconsin-Madison, School of Medicine, \\ Madison, WI; \\ 'United Network for Organ Sharing (UNOS), Richmond, \\ VA; \\ e University Renal Research and Education Association, \\ Ann Arbor, Ml; \\ f University of Michigan Health System, Ann Arbor, MI \\ *Corresponding author: Dixon B. Kaufman, \\ d-kaufman2@northwestern.edu
}

Over the past decade, immunosuppression therapy has undergone striking changes in the scale and pace by which new immunosuppressive molecules and antibodies have become incorporated into daily transplant medicine. An organ-by-organ review of data reveals several trends. The highest use of induction therapy (over $70 \%$ of patients) was reported for simultaneous pancreas kidney (SPK) and pancreas after kidney (PAK) transplants in 2002; use of induction therapy was less common in liver transplants (only $18 \%$ ). Corticosteroids served as discharge maintenance immunosuppression in over $87 \%$ of the recipients of kidney, SPK, PAK and thoracic transplants, and in over $\mathbf{7 0 \%}$ of pancreas transplant alone (PTA) recipients. Corticosteroid use in intestine transplants was reported in $64 \%$ of recipients in 2002 . A shift in the calcineurin inhibitor used for maintenance immunosuppression from cyclosporine to tacrolimus for the majority of patients had occurred for kidney, PAK, SPK, PTA, liver, lung, and heart-lung by 2001 . For heart transplants, cyclosporine remained the calcineurin

Notes on Sources: The articles in this report are based on the reference tables in the 2003 OPTN/SRTR Annual Report, which are not included in this publication. Many relevant data appear in the figures included here; other tables from the Annual Report that serve as the basis for this article include the following: Tables 5.6a-d, 6.6a-d, 7.6a-d, 8.6a-d, 9.6a-d, 10.6a-d, 11.6a-d, 12.6a-d, and 13.6a-d. All of these tables are also available online at http://www.ustransplant.org.

Funding: The Scientific Registry of Transplant Recipients (SRTR) is funded by contract \#231-00-0116 from the Health Resources and Services Administration (HRSA). The views expressed herein are those of the authors and not necessarily those of the US Government. This is a US Government-sponsored work. There are no restrictions on its use. inhibitor of choice; tacrolimus remained the predominant calcineurin inhibitor agent for intestine (since 1994). Use of antibody treatment for rejection during the first post-transplant year for most organs declined. Short-term outcomes have improved, based on the observation that rates of rejection within the first year post-transplant have diminished.

Key words: Antirejection treatment, immunosuppression, induction therapy, maintanence immunosuppression, SRTR, transplantation

\section{Introduction}

This article analyzes solid organ transplantation over the 10-year time span of 1993-2002. This period has been distinguished by marked changes in the clinical practice of transplantation in general, and in immunosuppressive strategies in particular. Certain aspects of the evolutionary changes of immunotherapeutics are captured quite nicely by the OPTN/SRTR (Organ Procurement \& Transplant Network/Scientific Registry of Transplant Recipients) data. Particularly strong components include the scale and pace by which the new immunosuppressive molecules and antibodies have become incorporated into the daily activities of transplant medicine. A careful organ-by-organ review of the data indicates how much has changed over the 10-year span beginning in 1993.

Regarding the use of maintenance immunosuppression, practice patterns indicate a strong role of calcineurin inhibitor-based immunosuppression. Yet this was occurring during a time when the availability of generic versions of cyclosporine was expanding and the collective use of cyclosporines, generally, was rapidly diminishing. In its place has been a sustained transition to the use of tacrolimus. Similar trends were also observed with the antimetabolites azathioprine and mycophenolate mofetilthe latter being used in the vast majority of transplant recipients. Recent years have seen the advent of the novel agent rapamycin, although its use has not mimicked the rapid and consistently successful incorporation of tacrolimus and mycophenolate mofetil. This may be indicative of uncertainty regarding its role in maintenance immunosuppression in organ transplantation at this juncture.

One practice trend in the use of maintenance immunotherapy that is not clearly elucidated by the data is the 
intensity of the immunosuppression. In one respect, it could be concluded that overall intensity has increased. Evidence of this viewpoint could be derived from increased use of the potent agents tacrolimus, mycophenolate mofetil, and rapamycin. From another perspective, there are clues that efforts are ongoing with aims of immunosuppression minimization. A hint of that is suggested by the subtle changes in the reduced application of chronic corticosteroid therapy. With more detailed data a more precise assessment could be deduced of the relative dosing schedules of the individual agents. Unfortunately, one of the limitations of the data is that it is relatively qualitative in nature. Specifics regarding the pharmacological exposure of the recipients to the individual agents are understandably beyond the SRTR aims at this time.

Regarding antibody-based induction therapy, practice patterns indicate that its use and intensity are rising each year. There are consistent trends indicating a switch to T-cell depleting agents, especially rabbit antithymocyte globulin. The lack of quantitative data regarding the actual dosing and duration of therapy, however, does not allow trends to be determined in that context. Use of alemtuzumab (Campath ${ }^{\circledR}$ ILEX Pharmaceuticals, San Antonio, TX) induction therapy (as an alternative T-cell depleting agent) and rituximab (Rituxan ${ }^{\circledR}$ Genentech, South San Francisco, CA) immunotherapy (used in antibody desensitization protocols) are beginning to be incorporated into practice, but have not reached a degree of penetration to be meaningful from a macroscopic viewpoint.

What follows is an examination of general practice trends in the use of maintenance and induction therapy being applied to kidney, pancreas, kidney-pancreas, liver, intestine, heart, heart-lung, and lung transplant recipients. It is the most complete, detailed, and up-to-date analysis of its kind available. Importantly, it is only through the collective efforts of the transplant community that this information is made available to transplant professionals and the multiple constituents with an interest in the field.

Unless otherwise noted, the statistics in this article come from reference tables in the 2003 OPTN/SRTR Annual Report. Two companion articles in this report, 'Transplant data: sources, collection, and caveats' and 'Analytical approaches for transplant research', explain the methods of data collection, organization, and analysis that serve as the basis for this article $(1,2)$. Additional detail on the methods of analysis may be found in the reference tables themselves or in the Technical Notes of the OPTN/SRTR Annual Report, both available online at http://www.ustransplant.org.

\section{Kidney Transplantation}

Trends in induction therapy in kidney transplantation The period from 1993 to 2002 has seen a gradual but definitive shift toward routine use of antibody induction therapy.

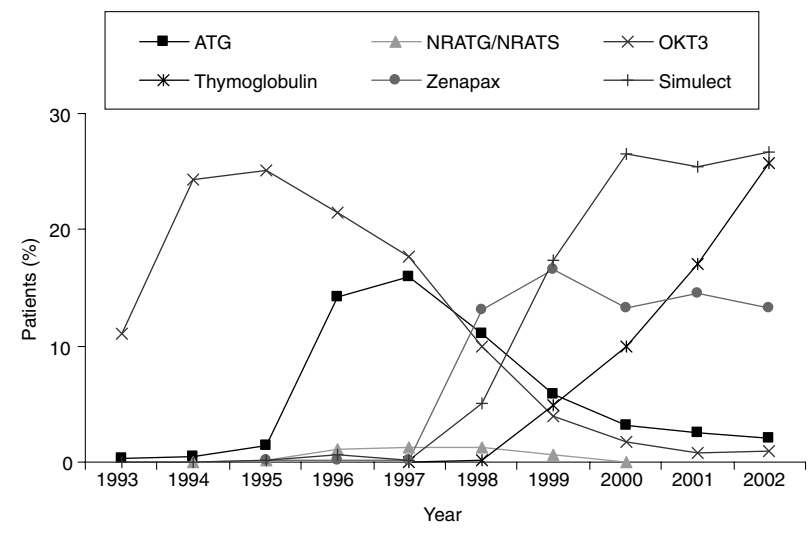

Source: 2003 OPTN/SRTR Annual Report, Table 5.6a.

Figure 1: Trends in kidney transplantation induction immunosuppression, 1993-2002.

In 1993, 11\% of patients undergoing kidney transplantation received antibody induction; by 2002 the proportion of kidney recipients who received antibody induction therapy was $65 \%$.

In addition to the increased use of antibody preparations for induction, there has been a marked shift in the type of antibody preparation (Figure 1). Muromonab-CD3 (OKT3 ${ }^{\circledR}$ Orthobiotech, Bridgewater, NJ), which peaked in use in 1995 (administered to $25 \%$ of kidney transplant recipients) and accounted for virtually all the antibody used for induction, had fallen to $1 \%$ by 2002 . Similar trends were observed for equine antithymocyte globulin (ATGAM ${ }^{\circledR}$ Pharmacia \& Upjohn, Kalamazoo, MI). Equine antithymocyte globulin, which had peaked in use in 1997 (received by $16 \%$ of kidney transplant recipients), had fallen to $2 \%$ by 2002 . The overwhelming majority of patients receiving antibody induction in 2002 received agents not available in 1993. These new agents included rabbit antithymocyte globulin (Thymoglobulin ${ }^{\circledR}$ SangStat Medical Corp., Fremont, CA), which accounted for $26 \%$ of patients, basiliximab (Simulect ${ }^{\circledR}$ Novartis, East Hanover, NJ), which accounted for $27 \%$, and daclizumab (Zenapax ${ }^{\circledR}$ Roche, Nutley, NJ), which accounted for $13 \%$ of patients. It is interesting to note that although daclizumab received FDA approval prior to basiliximab, basiliximab very quickly became the most commonly used induction agent shortly after its approval by the FDA. This may be related to the smaller number of doses (two) associated with its approved use and the ability to give both doses during the initial hospitalization at the time of transplantation.

Induction therapy was used less frequently in recipients of kidneys from living versus deceased donors, but additional SRTR analysis showed that the difference was small. In 2002, the proportion of living and deceased donor recipients receiving induction therapy was $62 \%$ and $67 \%$, respectively. With respect to the type of 
induction agent used in recipients of kidneys from deceased donors, rabbit antithymocyte globulin was used most commonly (29\%), followed by basiliximab (27\%). The type of induction agent used most commonly, however, in recipients of kidneys from living donors was basiliximab (27\%), followed by rabbit antithymocyte globulin (21\%).

\section{Trends in maintenance immunosuppression therapy prior to discharge in kidney transplantation}

Calcineurin inhibitors. In 1993, 95\% of patients undergoing kidney transplantation received cyclosporine; only $2 \%$ received tacrolimus (the latter had not yet been approved by the FDA) (Figure 2). The trend over the next 10 years has been a gradual but inexorable decline in the use of cyclosporine, down to $30 \%$ in 2002. Most of the cyclosporine used has been Neoral ${ }^{\circledR}$ (Novartis, East Hanover, NJ) 22\% and Gengraf ${ }^{\circledR}$ (Abbott, Abbott Park, IL) 7\%. Sandimmune ${ }^{\circledR}$ (Novartis, East Hanover, NJ) accounted for $1 \%$ and Eon ${ }^{\circledR}$ (Eon Labs, Laurelton, NY) for $0.2 \%$. Tacrolimus use has increased steadily as cyclosporine use has decreased (Figure 2). In 2002, tacrolimus was used in $63 \%$ of kidney transplant recipients. The reasons for this conversion are most likely related to multicenter trial data that have suggested lower rates of acute rejection and lower rates of steroidresistant rejection associated with tacrolimus $(3,4)$. Cosmetic issues, i.e. the absence of hirsutism with tacrolimus, have also played a role.

Antimetabolites and rapamycin. In 1993, azathioprine, the only routinely available antimetabolite, was used in $86 \%$ of kidney transplant recipients; by 2002 this had decreased to $2 \%$ (Figure 2). Mycophenolate mofetil, which was approved by the FDA in 1995, has seen its use increase to $79 \%$ of patients undergoing kidney transplantation. This high percentage rate has remained relatively constant over the last several years. In all, $81 \%$ of kidney recipients received some sort of antimetabolite in 2002. Rapamycin was received by $15 \%$ of the patients in 2002 . This was
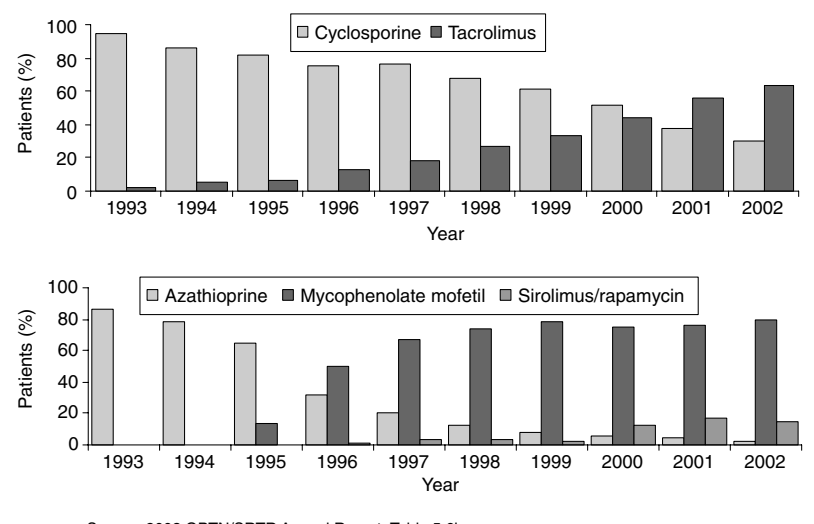

Source: 2003 OPTN/SRTR Annual Report, Table 5.6b.

Figure 2: Trends in kidney transplant maintenance immunosuppression prior to discharge, 1993-2002. down slightly from $17 \%$ in 2001 . The most popular combination regimen in recent years has included tacrolimus and mycophenolate mofetil, which has been utilized in the majority of patients undergoing kidney (or pancreas) transplantation. This combination has been shown to be effective in several single and multicenter prospective randomized trials (4-6).

Corticosteroids. In 1993, 99\% of patients received corticosteroids, either in the form of intravenous methylprednisone or prednisone. In 2002, 91\% of patients received corticosteroids, suggesting that there is a growing percentage of patients who are receiving either steroid avoidance or near avoidance protocols after kidney transplantation (7-9).

\section{Trends in maintenance immunosuppression therapy for the first year in kidney transplantation}

Calcineurin inhibitors. The data in this section mirror the data described in the previous section. Cyclosporine was used in $96 \%$ of kidney transplant recipients in 1992. This had decreased to $39 \%$ by 2001 . In 2001, Neoral ${ }^{\circledR}$ accounted for the vast majority of cyclosporine use (31\%), Gengraf ${ }^{\circledR}$ accounted for $9 \%$, Sandimmune ${ }^{\circledR} 3 \%$, and Eon ${ }^{\circledR}$ $0.3 \%$. Tacrolimus, which had not yet been approved in 1992, accounted for $3 \%$ of kidney transplant patients; by 2001 this figure had increased to $64 \%$, probably for the reasons discussed above.

Antimetabolites and rapamycin. The use of antimetabolites remained high in 2001 at $83 \%$ in kidney transplant recipients, however this was down somewhat from 1999, when it was $89 \%$. Azathioprine usage decreased from $87 \%$ in 1992 to $6 \%$ in 2001, and mycophenolate mofetil increased from $1 \%$ in 1992 to $80 \%$ in 2001. Rapamycin use increased from $0 \%$ in 1992 to $21 \%$ in 2001 ; virtually all this increase has occurred since its FDA approval in 1998.

Corticosteroids. Corticosteroids again remain in use in virtually all patients undergoing kidney transplantation; this percentage has fallen very slightly from $99.5 \%$ in 1992 to $96 \%$ in 2001 , suggesting that there is a small but growing percentage of patients who are no longer receiving corticosteroids.

\section{Trends in antirejection treatment in kidney transplantation}

The first important observation to be made about antirejection treatment is that the need for it has decreased dramatically. In 1992, the percentage of kidney transplant recipients undergoing antirejection treatment was 38\%, by 2001 it was 17\% (Figure 3). In terms of therapies for rejection, the overwhelming majority of cases were treated with corticosteroids, and this percentage has changed little over the years, remaining at $81 \%$ in 1992 and in 2001. Antibody therapy use has decreased over the years, from $53 \%$ in 1992 to $37 \%$ in 2001 . The specific antibodies used to 


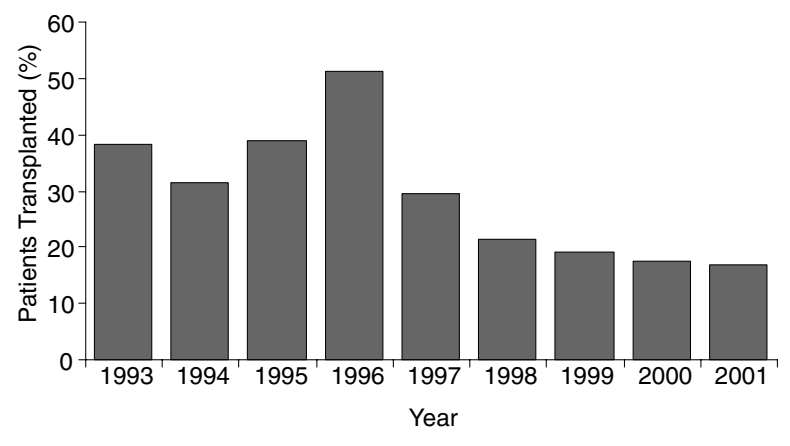

Source: 2003 OPTN/SRTR Annual Report, Table 5.6d.

Figure 3: Trends in incidence of rejection at 1 year in kidney transplant recipients, 1993-2001.

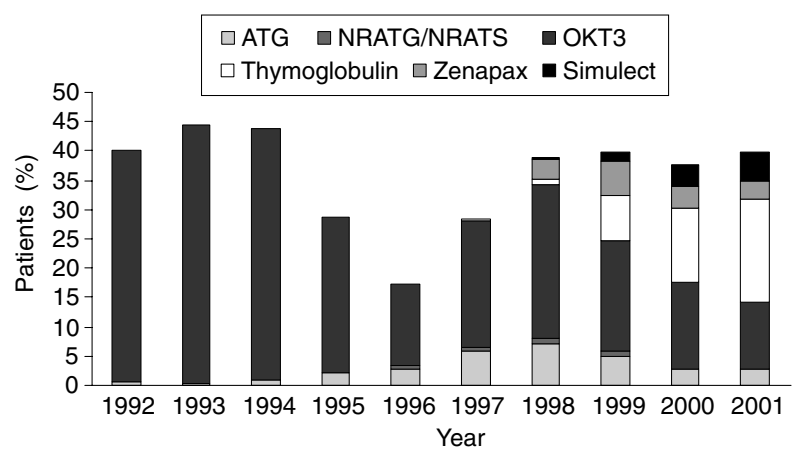

Source: 2003 OPTN/SRTR Annual Report, Table 5.6d.

Figure 4: Trends in antibody therapy for rejection episodes following kidney transplantation.

treat rejection, however, have changed dramatically (Figure 4). Antilymphocyte globulin (ALG), which peaked at $21 \%$ in 1993, has disappeared from practice. MuromonabCD3, which peaked at $44 \%$ in 1993 , is now down to $11 \%$. The use of rabbit antithymocyte globulin, which was $0 \%$ in 1993, is now up to $18 \%$. Interestingly, in 2001, the interleukin-2 (IL-2) receptor antagonists daclizumab and basiliximab were used in $3 \%$ and $5 \%$ of patients, respectively, as antirejection therapy, although there are little data supporting their use.

\section{Pancreas Transplantation}

\section{Trends in induction therapy in pancreas transplantation}

The use of induction therapy has been shown to significantly improve pancreas graft survival rates in several subgroups. According to data from the International Pancreas Transplant Registry, the use of induction therapy in simultaneous pancreas-kidney (SPK) transplant recipients with systemic venous-enteric exocrine drainage significantly improves pancreas graft survival rates $(10,11)$. Interestingly, pancreas graft survival is not improved with induction therapy in the subgroups with portal venous-enteric or bladder drainage. Furthermore, SPK transplant recipients who receive induction therapy benefit from a reduced incidence and severity of biopsy-confirmed, treated, acute kidney rejection episodes. In recipients given anti-T-cell depleting induction agents, however, there was also a statistically significantly higher rate of CMV viremia/syndrome, especially in the subgroup of recipients who received organs from CMV serologically positive donors (12). For the numerically smaller groups of solitary pancreas recipients (pancreas after kidney (PAK) and pancreas transplant alone (PTA)), the addition of induction therapy is associated with a clinically significant improvement in pancreas graft survival rates. In the PAK category, the 3-year actuarial pancreas graft survival with induction therapy is $74 \%$ vs. $64 \%$ without. In the PTA category, the 1-year functional survival rate for recipients with induction therapy was $86 \%$ vs. $74 \%$ without.

The use of antibody induction in pancreas transplant recipients has been relatively more common than in kidney transplantation. In SPK recipients, induction was used in $74 \%$ of patients in 2002. As in kidney transplantation, the specific agents have changed dramatically (Figure 5), so that while muromonab-CD3 was used in $56 \%$ of patients in 1995 , use had decreased to $0.9 \%$ by 2002 . Similarly, while equine antithymocyte globulin was used in $30 \%$ of cases undergoing SPK in 1997, use had decreased to $3 \%$ by 2002. Between 1993 and 2002, the use of rabbit antithymocyte globulin increased from $0 \%$ to $37 \%$; the use of daclizumab increased from $0 \%$ to $16 \%$; and the use of basiliximab increased from $0 \%$ to $25 \%$. In PAK and PTA patients, the same trends are observed. In PAK, no antibody induction was used in 1993, but in only $28 \%$ of cases in 2002 was antibody induction not used. Most of the antibody induction in 2002 was with rabbit antithymocyte globulin (51\%), daclizumab (26\%), and basiliximab (8\%).

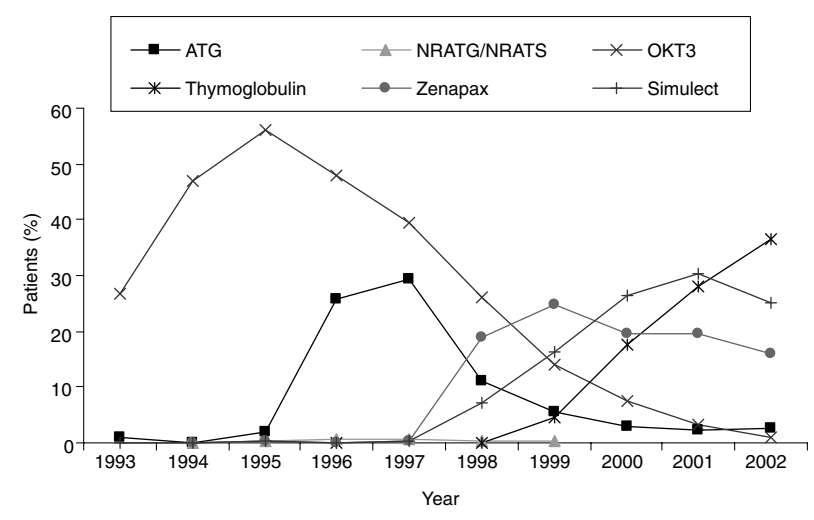

Source: 2003 OPTN/SRTR Annual Report, Table 8.6a.

Figure 5: Trends in simultaneous kidney-pancreas transplantation induction immunosuppression, 1993-2002. 
Similarly, in PTA patients, antibody induction was not used in $33 \%$ of patients in 2002; $58 \%$ received rabbit antithymocyte globulin, $34 \%$ received daclizumab, and $5 \%$ received basiliximab.

\section{Trends in maintenance immunosuppression therapy prior to discharge in pancreas transplantation}

Calcineurin inhibitors. The decrease in the use of cyclosporine between 1993 and 2002 has been even more pronounced in kidney-pancreas transplants (Figure 6). In 1993, 98\% of patients received cyclosporine; this was down to $9 \%$ in 2002 , with $7 \%$ receiving Neoral ${ }^{\circledR}, 2 \%$ receiving Gengraf ${ }^{\circledR}, 0.7 \%$ receiving Sandimmune ${ }^{\circledR}$, and $0.2 \%$ receiving Eon ${ }^{\circledR}$. Tacrolimus usage increased to $87 \%$ of SPK recipients in 2002, up from 0\% in 1993. Similar findings have been noted in both PAK and PTA patients; tacrolimus use increased from 0\% to $86 \%$ between 1993 and 2002 in PAK patients and from 10\% to $95 \%$ in PTA patients.

Antimetabolites and rapamycin. As in kidney-pancreas transplants, antimetabolites continued to be used in the vast majority of patients, although this percentage decreased from 95\% in 1993 to $80 \%$ in 2002 (Figure 6). Azathioprine usage fell from $95 \%$ to $0.9 \%$, while mycophenolate mofetil has increased from $0 \%$ to $79 \%$; this is down somewhat from 1999, where $88 \%$ of SPK patients received mycophenolate mofetil, and probably reflects the increased use of rapamycin. Rapamycin use in SPK patients increased from $0 \%$ in 1993 to as high as $20 \%$ in 2001; in 2002 it was down to 18\%. In PAK patients, the use of antimetabolites decreased from 100\% to 74\%. Azathioprine usage decreased from $100 \%$ to $0.3 \%$. Mycophenolate mofetil use increased from $0 \%$ to $74 \%$, but this represents a decrease from a peak of $92 \%$ in 1997 . In PAK patients, the use of rapamycin increased from $0 \%$ to a peak of $21 \%$ in 2001 , down to $19 \%$ in 2002 . In PTA patients, antimetabolite usage decreased from $87 \%$ in 1993 to $66 \%$ in 2002 . Azathioprine usage again virtually disap-
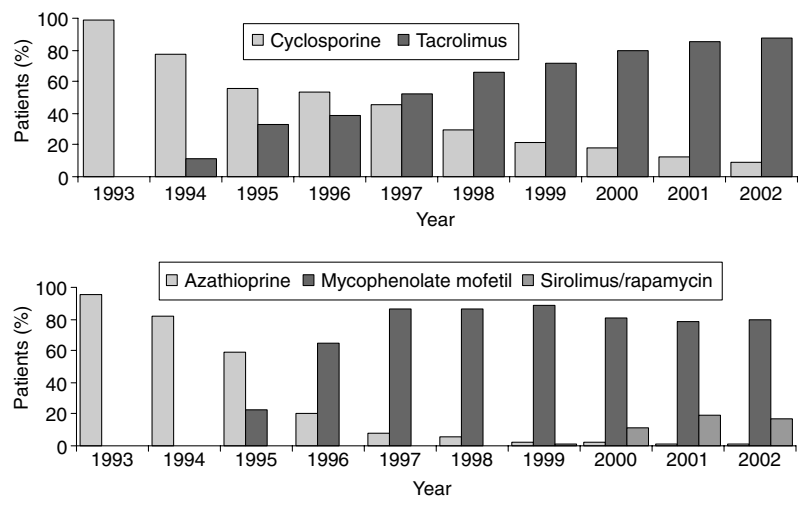

Source: 2003 OPTN/SRTR Annual Report, Table 8.6b.

Figure 6: Trends in SPK transplant maintenance immunosuppression prior to discharge, 1993-2002. peared, decreasing from $87 \%$ to $1 \%$, and mycophenolate mofetil usage increased from $0 \%$ to $64 \%$, the latter representing a decrease from $89 \%$ in 1997. In PTA patients, rapamycin usage increased steadily from $0 \%$ in 1993 to $16 \%$ in 2002 .

Corticosteroids. Corticosteroids were used in $99 \%$ of patients undergoing SPK in 1993; this has decreased over the last couple of years to $87 \%$ in 2002, reflecting an increased use of steroid-free protocols (13). In PAK patients, the data from the early 1990s seem somewhat incomplete, but the percentages from 1997 and 1998, with 95\% and 99\% receiving steroids, decreased slightly to $89 \%$ by 2002 . For PTA patients, the same trends were observed.

\section{Trends in maintenance immunosuppression therapy for the first year in pancreas transplantation}

Calcineurin inhibitors. The change in the use of calcineurin inhibitors has again been more marked in kidney-pancreas transplants. Cyclosporine use decreased from $99.8 \%$ to $16 \%$ between 1992 and 2001; most of this again was with Neoral ${ }^{\circledR} 12 \%$, Gengraf ${ }^{\circledR} 3 \%$, Sandimmune ${ }^{\circledR} 2 \%$, and Eon $^{\circledR}$ 0.4\%. In SPK recipients, tacrolimus use rose from $0.5 \%$ in 1992 to $89 \%$ in 2001 . The findings in PAK and PTA patients were even more dramatic. In PAK patients, cyclosporine use decreased from $100 \%$ in 1992 to $15 \%$ in 2001; tacrolimus use increased from 0\% to 93\%. In PTA patients, cyclosporine use decreased from $89 \%$ to $8 \%$ between 1992 and 2001, and tacrolimus use increased from $11 \%$ in 1992 to $99 \%$ in 2001.

Antimetabolites and rapamycin. Antimetabolite use decreased from 99\% in 1992 to $79 \%$ in 2001 in kidneypancreas transplants. There was a shift from azathioprine, which decreased from $99 \%$ to $2 \%$ during this time period, to mycophenolate mofetil, which went up from $0 \%$ to $78 \%$. The latter number is a decrease from 1998, where 92\% of SPK patients received mycophenolate mofetil; it probably reflects the increasing use of rapamycin. In PAK and PTA patients, the same trends are present. There has been a steady increase in the use of rapamycin in SPK patients, increasing from $0 \%$ to $27 \%$ between 1992 and 2001. This has been mirrored in PAK patients, rapamycin use has gone up from $0 \%$ to $29 \%$. In PTA patients, rapamycin use has increased from $0 \%$ to $15 \%$ (the latter, however, presents a decrease from 2000, where $25 \%$ of PTA patients received rapamycin).

Corticosteroids. Corticosteroid use in SPK patients decreased slightly from $100 \%$ in 1992 to $92 \%$ in 2001, reflecting an increased use of steroid-weaning protocols $(14,15)$. Steroid use, however, has remained relatively stable in PAK patients and is almost as high in PTA patients.

\section{Trends in antirejection treatment in pancreas transplantation}

As in kidney transplantation, there has been a marked reduction in the need for antirejection therapy. From 1992 to 


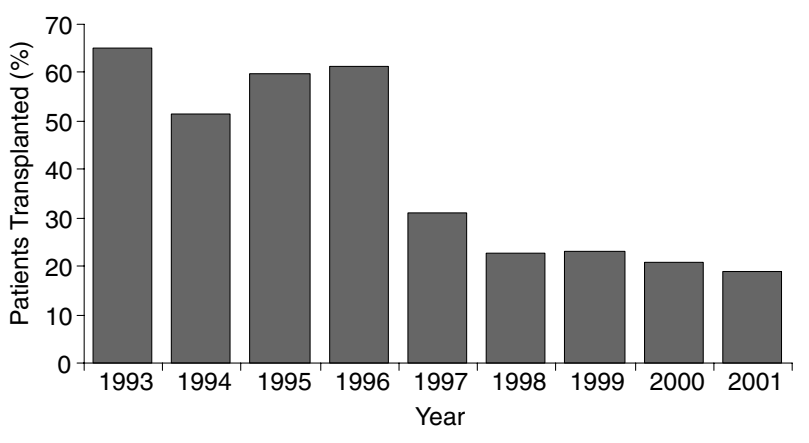

Source: 2003 OPTN/SRTR Annual Report, Table 8.6d.

Figure 7: Trends in incidence of rejection at 1 year in simultaneous kidney-pancreas transplant recipients, 1993-2001.

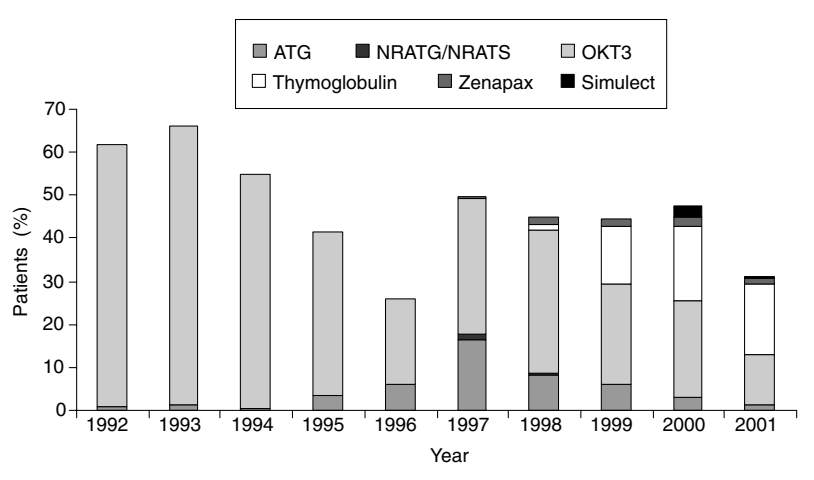

Source: 2003 OPTN/SRTR Annual Report, Table 8.6d.

Figure 8: Trends in antibody therapy for rejection episodes following simultaneous kidney-pancreas transplantation.

2001, the incidence of rejection in SPK patients declined from $66 \%$ to $19 \%$, in PAK patients from $45 \%$ to $14 \%$, and in PTA patients from $54 \%$ to $13 \%$ (Figure 7). Antibody use decreased from $73 \%$ in 1992 to $29 \%$ in 2001 in SPK patients, and again the type of agents changed from muromonab-CD3 and equine antilymphoblast globulin $(61 \%$ and $25 \%$, respectively, in 1992$)$ to rabbit antithymocyte globulin and muromonab-CD3 (accounting for $17 \%$ and $12 \%$ of patients, respectively, in 2001) (Figure 8). Corticosteroid usage has remained stable at just over $85 \%$. In PAK and PTA patients, antibody therapies were used in a somewhat higher percentage of patients by 2001 (63\% in PAK patients, 62\% in PTA patients). The nature of these agents has also changed, as in the case of SPK.

\section{Liver Transplantation}

Trends in induction therapy in liver transplantation According to OPTN/SRTR data, between 1993 and 2001, the use of induction agents varied from $10 \%$ to $15 \%$ of recorded cases; by 2002 this figure had crept up to

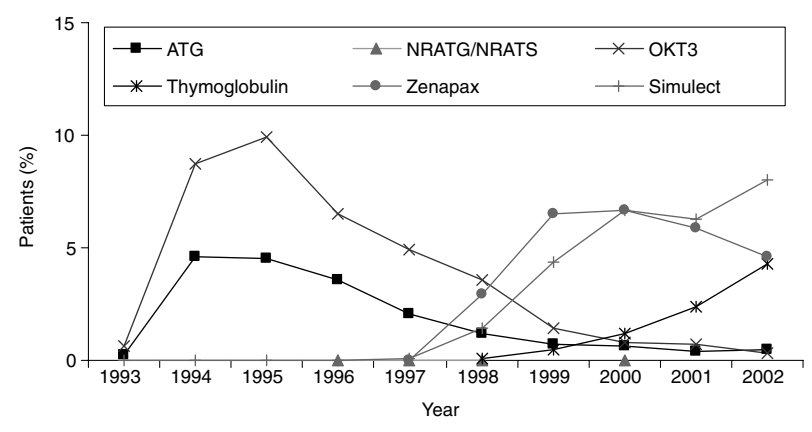

Source: 2003 OPTN/SRTR Annual Report, Table 9.6a.

Figure 9: Trends in liver transplantation induction immunosuppression, 1993-2002.

$18 \%$. During the last 10 years, the induction agents of choice have undergone gradual change. Earlier in the era, muromonab-CD3 was the predominant agent used, reaching a zenith in 1995 when $10 \%$ of recipients received it. Since then, the use of muromonab-CD3 has declined to almost nil, whereas the use of monoclonal anti-IL2 receptor agents has increased, so that by $2002,13 \%$ of recipients received either daclizumab or basiliximab (Figure 9). There also has been a modest resurgence in the use of rabbit antithymocyte globulin.

The reasons behind the gradual increased use of induction agents over the past 5 years are not clear. There are few studies to support or refute the use of antilymphocyte induction therapy in primary or repeated liver transplantation. One possible explanation is the introduction in 2002 of MELD-based allocation, in which liver transplantation candidates with renal failure receive priority; if such patients are being transplanted more quickly, it may reflect increased use of such agents as calcineurin inhibitor sparing agents in this population.

\section{Trends in maintenance immunosuppression therapy prior to discharge in liver transplantation}

Serial data from 1993 through 2002 show some consistent patterns of immunosuppressant use prior to discharge. Throughout the period, liver allograft recipients were managed on multiple drug regimens based on corticosteroids and calcineurin inhibitors. Consistently, at least $50 \%$ of recipients received an antimetabolite. During the period for which data have been collected, there have been minor and inconsistent variances in the prevalence of corticosteroid use, so that $90 \%$ of recipients were discharged on corticosteroids in 2002, compared with $95 \%$ in 1993 or $88 \%$ in 1995.

Calcineurin inhibitors. In contrast, there have been striking shifts in drug selection among the calcineurin inhibitors and antimetabolites (Figure 10). For example, whereas cyclosporine (in any of its formulations) was administered to $83 \%$ of recipients prior to discharge in 1993 , its rate of 

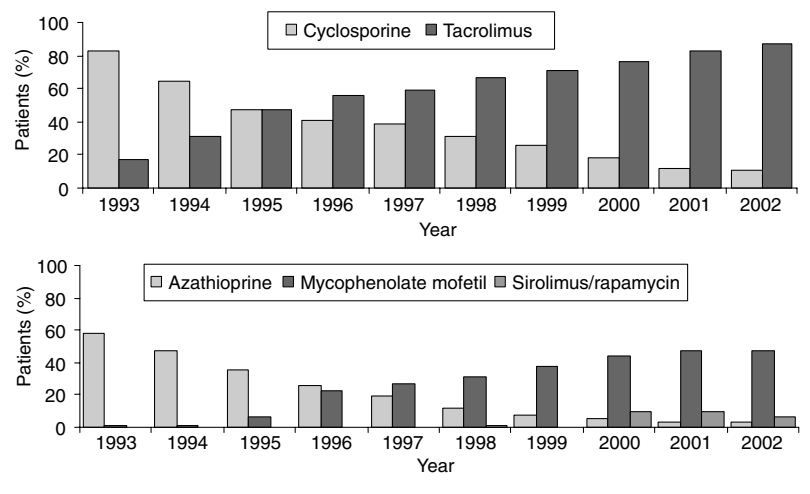

Source: 2003 OPTN/SRTR Annual Report, Table 9.6b.

Figure 10: Trends in liver transplant maintenance immunosuppression prior to discharge, 1993-2002.

use has declined every year since then to reach its current nadir of $10 \%$ in 2002 . Whether the newly introduced practice of monitoring the blood level 2 h (C2 monitoring) after ingestion rather than fasting trough levels will reverse this trend remains to be seen. The decline in use of cyclosporine has been mirrored by the increase in use of tacrolimus. Its use in immunosuppressant management prior to discharge increased every year from 1993 (18\%) to $2002(87 \%)$.

Antimetabolites and rapamycin. Similarly, azathioprine was the antimetabolite of choice in 1993, being administered to $58 \%$ of recipients. This rate of use declined annually until 2001, when it reached a nadir of $3 \%$. In 2002, azathioprine use showed a modest increase to $4 \%$. At the same time azathioprine declined, mycophenolate mofetil gained in use, from $0.6 \%$ in 1993 to $48 \%$ in 2002 (Figure 10). These data suggest, but do not prove, that mycophenolate mofetil has replaced azathioprine in circumstances where the managing physicians believe that an antimetabolite is required. Finally, there has been a small increase in the use of rapamycin in the early postoperative period. It was administered to $9 \%$ and $10 \%$ of recipients in 2000 and 2001, respectively. Curiously, this prevalence of use declined to $7 \%$ in 2002, perhaps on account of reported difficulties with early postoperative management, such as wound healing and integrity of the vascular anastomoses.

\section{Trends in maintenance immunosuppression therapy for the first year in liver transplantation}

Perhaps the most surprising aspect of the OPTN/SRTR data on maintenance immunosuppression between discharge and the end of the first year is the unchanging results for use of corticosteroids. Thus, 93\% of recipients in 1993 and $90 \%$ of recipients in 2001 received corticosteroids for their first postoperative year. This is curious because of the widespread discussion of steroid reduction and withdrawal after liver transplantation (16-18). There are a number of possible explanations for this discrepancy. First, practice may have changed since 2001; if so, more contemporary data will show less use of corticosteroids. Second, there may be a gap between actual practice and what is advocated in publications, with practice not changing as much as anticipated. Third, the presented data do not consider the doses of corticosteroids in use, which may have been reduced over the past 10 years, albeit without withdrawal. Finally, the interval from discharge to the end of year 1 may be too brief, and data on maintenance up to year 5 might be more indicative of steroid withdrawal. In any case, the present data do not confirm a major shift away from the use of corticosteroids for maintenance immunosuppression in the first year after liver transplantation.

The data on calcineurin inhibitors and antimetabolites in the first year mirror the data reported prior to discharge, namely: (i) the widespread use of tacrolimus where previously cyclosporine was the favored calcineurin inhibitor and (ii) the reduction in use of azathioprine and concomitant adoption of mycophenolate mofetil as the antimetabolite of choice. A small but significant percentage of patients also received rapamycin, the use of which grew from $0 \%$ in 1997 to $17 \%$ in 2001 . These data do not address specifically the combinations in use. Nevertheless, judging by these data, even in 2001, more that $80 \%$ of liver transplant recipients were receiving at least two immunosuppressives (both corticosteroids and tacrolimus) and approximately half were receiving three.

\section{Trends in antirejection treatment in liver transplantation}

The proportion of liver transplant recipients treated for rejection has diminished over the 10-year time span, 1992 (47\%) through 2001 (28\%). During the most recent time period (1996 through 2001), however, the rate remained nearly constant (32\% and 28\%, respectively) (Figure 11). With respect to treatment therapies for rejection, the majority of cases were treated with corticosteroids. The percentage changed very little over the years: 94\% in 1992

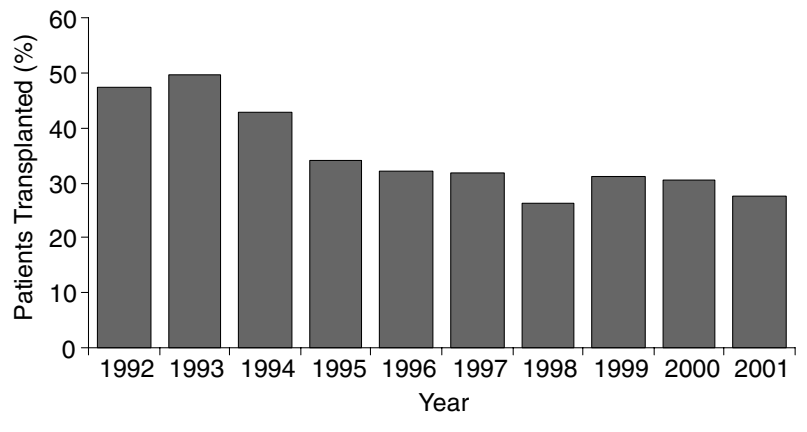

Source: 2003 OPTN/SRTR Annual Report, Table 9.6d.

Figure 11: Trends in incidence of rejection at 1 year in liver transplant recipients, 1992-2001. 


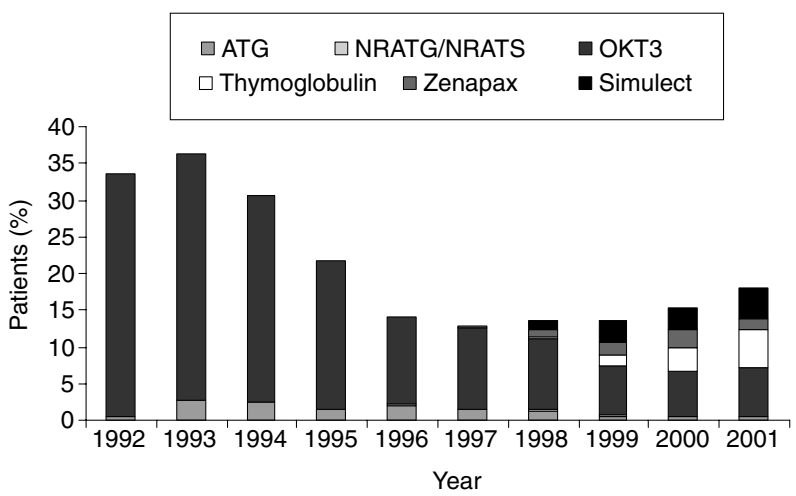

Source: 2003 OPTN/SRTR Annual Report, Table 9.6d.

Figure 12: Trends in antibody therapy for rejection episodes following liver transplantation.

to $89 \%$ in 2001 . Antibody therapy decreased slightly over the years, from $33 \%$ in 1992 to $17 \%$ in 2001 . The nature of the antibodies used to treat rejection changed dramatically (Figure 12). Muromonab-CD3, which peaked at 34\% in 1993, went down to $7 \%$ in 2001 . Rabbit antithymocyte globulin, for which usage was $0 \%$ in 1993, was used in $5 \%$ of cases in 2001 . Interestingly, the IL-2 receptor antagonists daclizumab and basiliximab were used in $2 \%$ and $4 \%$ of patients, respectively, as antirejection therapy in 2001, although there are little data supporting their use.

\section{Intestinal Transplantation}

\section{Trends in induction therapy in intestinal transplantation}

The numbers of intestinal transplants remain small, 107 in 2002. The data presented do not distinguish between combined liver-small bowel transplantation and isolated small bowel transplantation. In the past 5 years, induction chemotherapy has become more common. The prevalence has risen from $41 \%$ in 1998 to $57 \%$ in 2002. Rabbit antithymocyte globulin, basiliximab, and daclizumab were all used, without one agent establishing clinical predominance.

\section{Trends in maintenance immunosuppression therapy prior to discharge in intestinal transplantation}

Calcineurin inhibitors. Exclusive and almost universal use of tacrolimus (96\% in 2002) has been the pattern prior to discharge throughout the period under review. Conversely, cyclosporine was used in no patients. Up to year 2000, there was a similar, almost universal use of corticosteroids. In the most recent 2 years, however, corticosteroid use prior to discharge has declined to $80 \%$ and $64 \%$, respectively. These data are difficult to explain, especially since a similar decline was not observed in corticosteroid use in the first year after discharge (see below).
Antimetabolites and rapamycin. Rapamycin was used prior to discharge in $19 \%$ of intestinal transplant recipients in 2002. This frequency had declined from a peak of $39 \%$ in 2000. Given the small numbers, it is difficult to infer the reasons for such changes in practice. The use of antimetabolite agents has been similarly inconsistent, and, most recently, no recipients were recorded as receiving azathioprine and only $11 \%$ received mycophenolate mofetil.

Trends in maintenance immunosuppression therapy for the first year in intestinal transplantation

The practice patterns for maintenance immunosuppression from discharge through the first year mirror those described above for transplant admission. Once again, tacrolimus was used in all recipients to the absolute exclusion of cyclosporine. This pattern has remained unchanged since 1996. Corticosteroids were also almost universal during this period, a pattern that has remained constant with minor variations since 1993.

The use of other agents is much less consistent over time. No clear trend regarding antimetabolites was discernable, with wide shifts from year to year. While recognizing the limited data, it appears that mycophenolate mofetil, which peaked at $57 \%$ in 1996, had fallen out of favor and was administered to only $3 \%$ of cases in 2001 . Rapamycin was recorded in 1999, 2000, and 2001 only. The frequency of its use has fluctuated widely.

\section{Trends in antirejection treatment in intestinal transplantation}

The proportion of intestine transplant recipients treated for rejection over the first post-transplant year has diminished over the 10-year time span, from 1992 (77\%) through 2001 (36\%). The data do not indicate the proportion of recipients with multiple rejection episodes. With respect to treatment therapies for rejection, virtually all recipients received corticosteroids as part of their antirejection therapy. Antibody therapy remained relatively constant: $48 \%$ in 1993 and $35 \%$ in 2001 . The nature of the antibodies used to treat rejection has not changed appreciably. In 1993, all antibody therapy was muromonab-CD3. It is still the most commonly used antibody for treating rejection. Rabbit antithymocyte globulin was used in 8\% of cases in 2001.

\section{Heart Transplantation}

\section{Trends in induction therapy in heart transplantation}

Trends for the use of induction therapy in heart transplantation have been similar to those seen in kidney transplantation, however there appears to be a lag time of roughly 2 years. This probably represents the general reluctance of thoracic organ transplant physicians to try newer therapies until safety and efficacy have been established in other organ groups. In general, over the last decade, there has been an increase in the percentage of patients receiving induction therapy, from $12 \%$ in 1993 to $45 \%$ in 2002 . This 


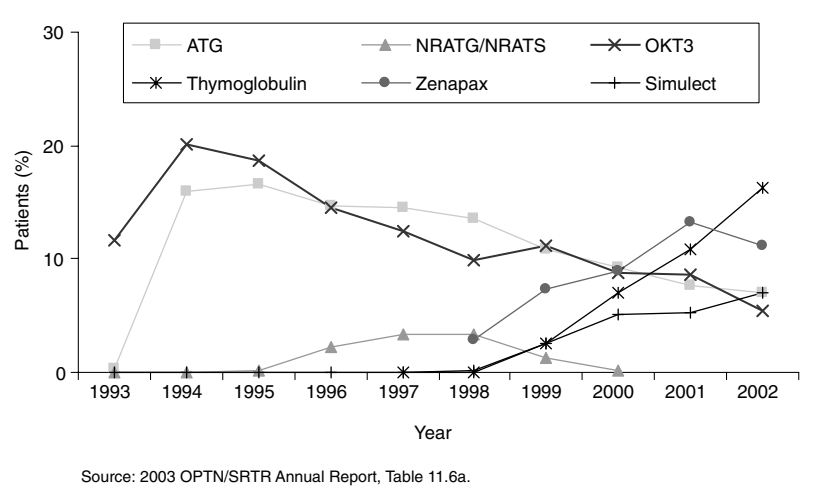

Figure 13: Trends in heart transplantation induction immunosuppression, 1993-2002.

may be explained, in part, by the fact that a higher risk population (i.e. patients presenting with more diabetes, renal dysfunction, and older age) is now being transplanted than a decade ago. There have been several obvious trends for specific agents (Figure 13). The most frequently used induction agents have been muromonab-CD3 and equine antithymocyte globulin; however, their use has rapidly fallen off in the last several years to $5 \%$ and $7 \%$, respectively. In contrast, the use of rabbit antithymocyte globulin has increased from $0 \% 4$ years ago to $16 \%$ in 2002. Likewise, the use of anti-IL-2 receptor antibodies (daclizumab and basiliximab) has increased from $0 \% 5$ years ago to $18 \%$ in 2002. The use of either rabbit antithymocyte globulin or an anti-IL-2 receptor antibody now appears to be the dominant strategy for induction in heart transplantation.

\section{Trends in maintenance immunosuppression therapy prior to discharge in heart transplantation}

From 1993 to 2002, there have been no major changes in the general scheme of immunosuppression therapy prior to discharge for heart transplant recipients. The basic therapeutic strategy for the majority of patients still includes a calcineurin inhibitor, an antimetabolite, and corticosteroids. There have been, however, some fairly dramatic changes in agents within each class.

Calcineurin inhibitors. Calcineurin inhibitor use at the time of discharge remains essentially $100 \%$ (Figure 14). Cyclosporine remains the most frequently used calcineurin inhibitor, although its use has fallen from $98 \%$ in 1993 to $61 \%$ in 2002 . Of those who received cyclosporine, approximately $46 \%$ received Neoral $^{\circledR}$ and $13 \%$ received Gengraf ${ }^{\circledR}$ (a small percentage, $3 \%$, received Sandimmune ${ }^{\circledR}$ ). The use of tacrolimus has increased proportionally to the decrease in cyclosporine use. Tacrolimus is now used in 39\% of heart transplant recipients at the time of discharge.

Antimetabolites and rapamycin. The use of antimetabolites (azathioprine and mycophenolate mofetil) at the time of discharge has remained fairly constant over the past decade,
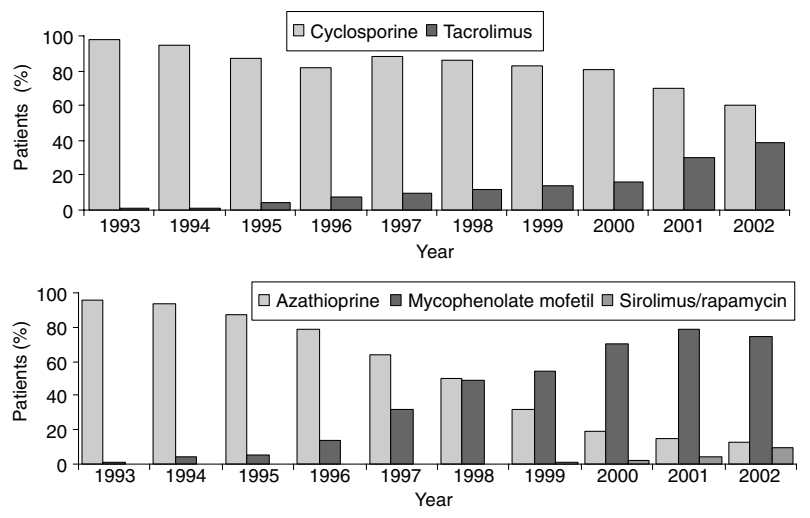

Source: 2003 OPTN/SRTR Annual Report, Table 11.6b.

Figure 14: Trends in heart transplant maintenance immunosuppression prior to discharge, 1993-2002.

ranging from $96 \%$ in 1993 to $84 \%$ in 2002 (Figure 14). Within this class of immunosuppressants, mycophenolate mofetil has assumed the dominant role over the past few years. In 2002, mycophenolate mofetil was used in $75 \%$ of heart transplant recipients at the time of discharge, while azathioprine use had fallen to $12 \%$. A slight fall-off in antimetabolite use over the past few years is probably explained by the increasing use of rapamycin, now used in $10 \%$ of heart transplant recipients at the time of discharge. Other agents, such as cyclophosphamide, are rarely used in heart transplantation.

Corticosteroids. Use of corticosteroids has fluctuated little in the past decade (a low of $94 \%$, a high of $97 \%$ ). In 2002, corticosteroids were used in $97 \%$ of heart transplant recipients at the time of discharge.

\section{Trends in maintenance immunosuppression therapy for the first year in heart transplantation}

In 2001, the most common maintenance therapy combination for heart transplant recipients within the first year after transplantation consisted of corticosteroids, cyclosporine or tacrolimus, and mycophenolate mofetil.

Calcineurin inhibitors. Therapy with cyclosporine or tacrolimus remains the cornerstone of maintenance immunosuppression in heart transplant recipients. Over the past 10 years, there has been a trend towards less use of cyclosporine and more use of tacrolimus. Cyclosporine use has decreased from 99\% in 1992 to $65 \%$ in 2001, with Neoral ${ }^{\circledR}$ being the most frequent formulation. The use of tacrolimus has greatly increased from $1 \%$ to $43 \%$ during the same time interval.

Antimetabolites and rapamycin. Although antimetabolites are still frequently used within the first year after heart transplantation, there has been a shift in practice over the last 10 years. The use of azathioprine dominated up until 
1996 (97\% in 1992, down to $15 \%$ in 2001). The use of mycophenolate mofetil now appears to be standard practice $(81 \%$ in 2001$)$ in heart transplantation. Overall, there appears to be a slight decline in the use of the standard antimetabolites as a class. In 1992, approximately $97 \%$ of heart transplant recipients received either azathioprine or mycophenolate mofetil. In 2001, the use of either azathioprine or mycophenolate mofetil fell to $95 \%$. Concurrent with this trend, there was an increase in use of other novel immunosuppressant agents. In 2001, approximately $8 \%$ of heart transplant recipients received rapamycin within the first year after transplantation.

Corticosteroids. The vast majority of heart transplant recipients are treated with corticosteroids as part of maintenance therapy during the first year after transplantation. Over the last 10 years, there has been no clear trend in the use of corticosteroids. In 2001, 91\% of heart transplant recipients received either prednisone or methylprednisolone.

\section{Trends in antirejection treatment in heart transplantation}

In the last decade, it appears that the rates for treatment of rejection within the first year after heart transplantation have not changed (39\% in 1992 and 38\% in 2001) (Figure 15). For patients who require therapy for rejection, the use of corticosteroids has remained between $88 \%$ and $92 \%$. During the last 10 years, the use of therapy with antibodies gradually decreased from $23 \%$ to $16 \%$. When antibody therapy was used, muromonab-CD3 remained the most frequently used preparation, although its use has declined. In 1993, muromonab-CD3 was used in $24 \%$ of cases of rejection, whereas in 2001 it was used in only $6 \%$. The use of equine antithymocyte globulin varied from $1 \%$ in 1992 to $7 \%$ in 1995 , although the use of this agent may be declining (4\% in 2001). The use of rabbit antithymocyte globulin has been steadily increasing over the past several years to $5 \%$ in 2001 . The use of anti-IL-2 receptor

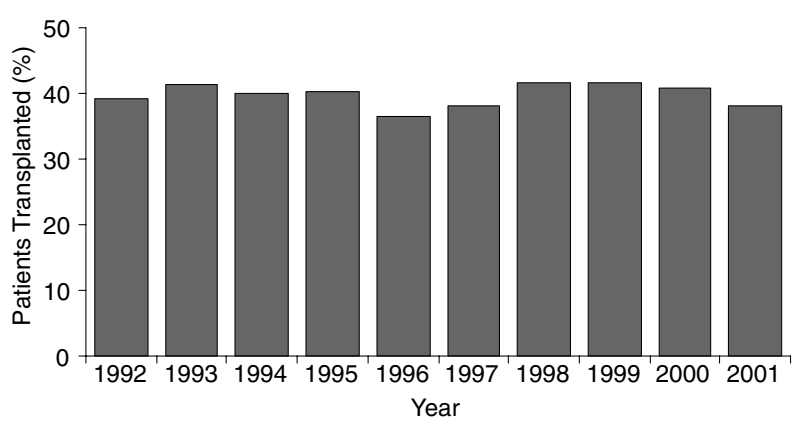

Source: 2003 OPTN/SRTR Annual Report, Table 11.6d.

Figure 15: Trends in incidence of rejection at 1 year in heart transplant recipients, 1992-2001.

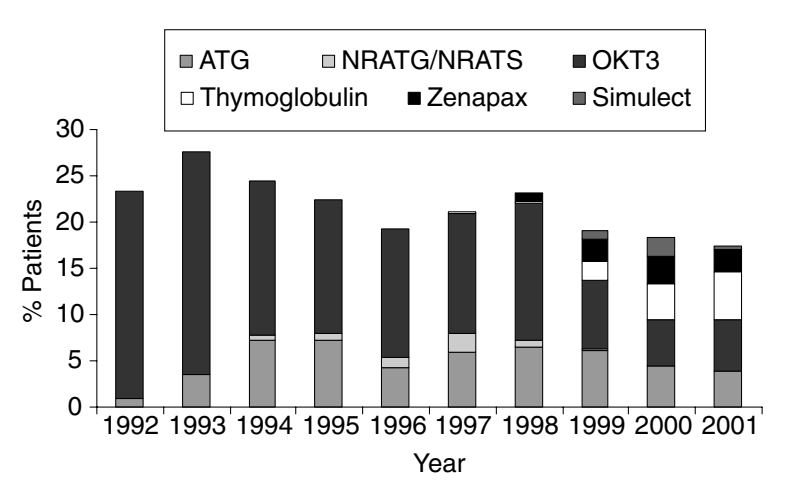

Source: 2003 OPTN/SRTR Annual Report, Table 11.6d.

Figure 16: Trends in antibody therapy for rejection episodes following heart transplantation.

antibodies for the treatment of rejection had increased to approximately $3 \%$ in 2001.

In summary, the treatment of rejection within the first year after heart transplantation remains based on corticosteroids, with a gradual decline in the use of antibody therapy noted over the past several years. When antibody therapy is used, there appears to be no consensus as to the specific preparation (Figure 16).

\section{Lung Transplantation}

\section{Trends in induction therapy in lung transplantation}

The trends for the use of induction therapy in lung transplantation have been similar to those seen in heart transplantation, with a general decrease seen in the use of muromonab-CD3 and equine antithymocyte globulin, and a concurrent increase in the use of rabbit antithymocyte globulin and anti-IL-2 receptor antibodies (daclizumab and basiliximab) (Figure 17). In general, over the last 10 years, there has been a gradual increase in the percentage of patients receiving induction therapy, from $3 \%$ in 1993 to $41 \%$ in 2002 . There have been several obvious trends for specific agents. The most frequently used induction agent over the last 10 years was equine antithymocyte globulin, peaking in 1995 at 23\%; however, in 2002, it was only reported in $7 \%$ of lung transplant recipients. The use of muromonab-CD3 peaked in 1994 at $6 \%$ but has now fallen to less than $0.6 \%$ in lung transplantation. In contrast, the use of rabbit antithymocyte globulin has increased from $0 \% 4$ years ago to $9 \%$ in 2002. Likewise, the use of anti-IL-2 receptor antibodies (daclizumab and basiliximab) has increased from $0 \%$ 6 years ago to $25 \%$ in 2002 . The use of either rabbit antithymocyte globulin or an anti-IL-2 receptor antibody now appears to be the dominant strategy for induction in lung transplantation. 
Dixon B. Kaufman et al.

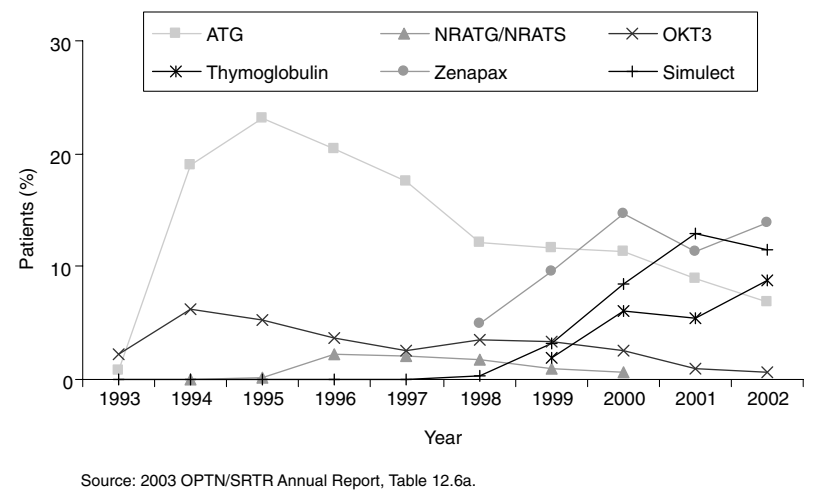

Figure 17: Trends in lung transplantation induction immunosuppression, 1993-2002.

\section{Trends in maintenance immunosuppression therapy prior to discharge in lung transplantation}

As in heart transplantation, from 1993 to 2002, there have been no major changes in the general scheme of immunosuppression therapy prior to discharge for lung transplant recipients. Within the certain specific classes of immunosuppressants, however, there have been dramatic shifts in practice.

Calcineurin inhibitors. Calcineurin inhibitor use at the time of discharge remains essentially $100 \%$. Cyclosporine is no longer the most frequently used calcineurin inhibitor. Cyclosporine fell from $91 \%$ in 1993 to $43 \%$ in 2002. Of those who received cyclosporine, most received Neoral ${ }^{\circledR}$. A small proportion received either Gengraf ${ }^{\circledR}$ or Sandimmune ${ }^{\circledR}$. In contrast, the use of tacrolimus increased substantially. Tacrolimus is now used in 57\% of lung transplant recipients at the time of discharge (Figure 18).
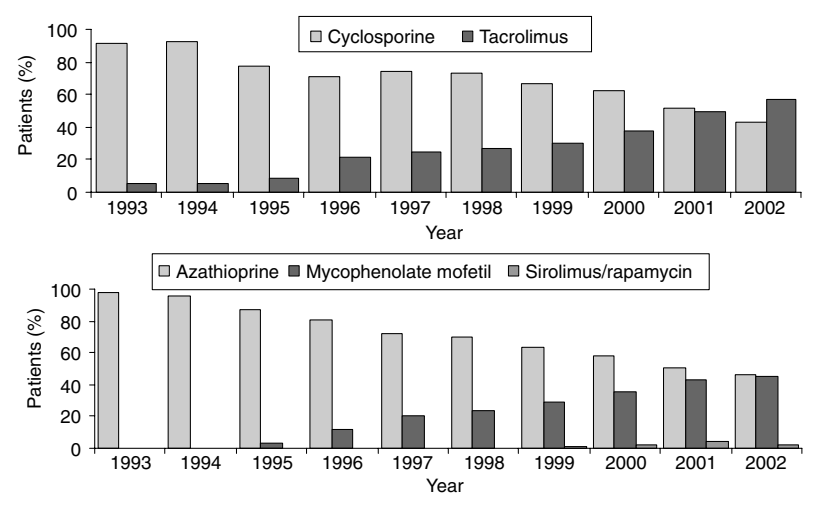

Source: 2003 OPTN/SRTR Annual Report, Table 12.6b

Figure 18: Trends in lung transplant maintenance immunosuppression prior to discharge, 1993-2002.
Antimetabolites and rapamycin. The use of antimetabolites (azathioprine and mycophenolate mofetil) at the time of discharge has remained fairly constant over the past 10 years, ranging from $98 \%$ in 1993 to $90 \%$ in 2002 . Although the use of azathioprine has declined, and the use of mycophenolate mofetil has proportionally risen, this has not occurred to the same extent as is seen in heart transplantation. In 2002, azathioprine was used in $46 \%$ of lung transplant recipients at the time of discharge and mycophenolate mofetil was used in $45 \%$ (Figure 18). The use of rapamycin has not, as of yet, played much of a role in lung transplantation. Peak use in 2001 was only 4\%. In 2002, rapamycin was only used in $2 \%$ of lung transplant recipients at the time of discharge. Other agents, such as cyclophosphamide, were rarely used in lung transplantation.

Corticosteroids. Use of corticosteroids fluctuated little in the past decade (a low of $97 \%$ and a high of $99 \%$ ). In 2002 corticosteroids were used in $99 \%$ of lung transplant recipients at the time of discharge.

\section{Trends in maintenance immunosuppression therapy for the first year in lung transplantation}

In 2001, the most common maintenance therapy combination for lung transplant recipients within the first year after transplantation consisted of corticosteroids, tacrolimus, and either azathioprine or mycophenolate mofetil.

Calcineurin inhibitors. As in heart transplantation, cyclosporine or tacrolimus remain the cornerstone of maintenance immunosuppression in lung transplant recipients. In the past decade, there was a trend toward less use of cyclosporine and more use of tacrolimus, to the point where tacrolimus now appears to be the agent of choice. Cyclosporine use has decreased from 93\% in 1992 to $46 \%$ in 2001 , with $\mathrm{Neoral}^{\circledR}$ being the most frequent formulation. The use of tacrolimus has increased from $7 \%$ to $69 \%$ during the same time interval.

Antimetabolites and rapamycin. Although antimetabolites are still frequently used within the first year after lung transplantation, there was a shift in practice over the last decade. The use of azathioprine declined from $97 \%$ in 1992 to $49 \%$ in 2001 . The use of mycophenolate mofetil concurrently increased from $0 \%$ to $52 \%$ over the same time period. Overall, there appeared to be a slight decline in the use of standard antimetabolites as a class. In 1992, approximately $97 \%$ of lung transplant recipients received azathioprine. In 2001, the use of either azathioprine or mycophenolate mofetil fell to $91 \%$. Concurrent with this trend, there was an increase in use of other novel immunosuppressant agents. In 2001, approximately $11 \%$ of lung transplant recipients received rapamycin within the first year after transplantation. Recent trends, however, show a decrease in use, perhaps related to the observation that airway anastomotic dehiscence is associated with use of rapamycin immediately after lung transplantation (19). 
Corticosteroids. The vast majority of lung transplant recipients are treated with corticosteroids as part of maintenance therapy during the first year after transplantation. Within the last decade, there has been no clear trend in the use of corticosteroids. In 2001, nearly $100 \%$ of lung transplant recipients received either prednisone or methylprednisolone.

\section{Trends in antirejection treatment in lung transplantation}

In the last decade, it appears that the rates for treatment of rejection within the first year after lung transplantation did not change substantially $(48 \%$ in 1992 and $45 \%$ in 2001) (Figure 19). For patients who required therapy for rejection, the use of corticosteroids has remained between $88 \%$ and $97 \%$.

During the last 10 years, the use of therapy with antibodies ranged from $9 \%$ to $18 \%$, with no clear trend. When antibody therapy was used, equine antithymocyte globulin was the most frequently used preparation, although its use declined from $10 \%$ in 1994 to $4 \%$ in 2001 . The use of muromonab-CD3 declined from 10\% in 1993 to $2 \%$ in 2001. The use of rabbit antithymocyte globulin has steadily increased over the past several years to $6 \%$ in 2001 . The use of anti-IL-2 receptor antibodies for the treatment of rejection was approximately $1 \%$ in 2001 .

In summary, the treatment of rejection within the first year after lung transplantation remains based on corticosteroids. The use of antibody therapy gradually increased between 1995 (10\%) and 2000 (18\%), however its usage decreased in 2001 (12\%). When antibody therapy is used, there appears to be no consensus as to the specific preparation (Figure 20).

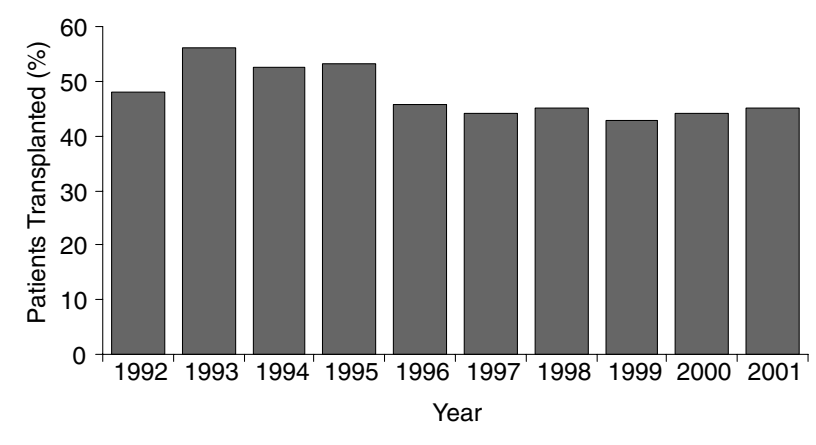

Source: 2003 OPTN/SRTR Annual Report, Table 12.6d.

Figure 19: Trends in incidence of rejection at 1 year in lung transplant recipients, 1992-2001.

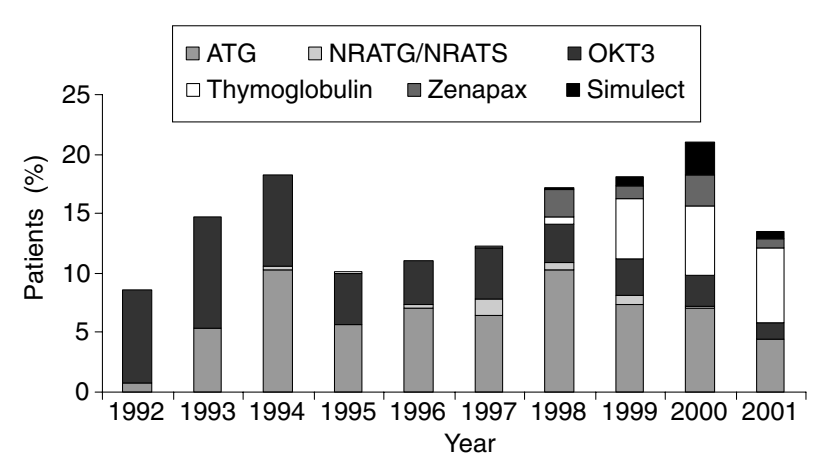

Source: 2003 OPTN/SRTR Annual Report, Table 12.6d.

Figure 20: Trends in antibody therapy for rejection episodes following lung transplantation, 1992-2001.

\section{Heart-Lung Transplantation}

\section{Trends in induction therapy in heart-lung transplantation}

The trends for the use of induction therapy in heart-lung transplantation have been somewhat erratic. The reason for this is that the overall number of en-bloc heart-lung transplants has decreased from a peak of 71 transplants in 1994 to as low as 27 in 2001 (32 in 2002). Thus, inference of trends in such small numbers is difficult. In general, over the last decade there has been a gradual increase in the percentage of patients receiving induction therapy, from $12 \%$ in 1993 to $59 \%$ in 2002 . The changes in practice, however, appear to be somewhat random. The use of equine antithymocyte globulin peaked in 1995 at 44\% and declined to $16 \%$ in 2002 . The use of muromonab-CD3 remained relatively stable (6\% in 2002). The use of rabbit antithymocyte globulin increased from $0 \% 4$ years ago to a peak in 2001 at 12\% (3\% in 2002). The use of anti-IL-2 receptor antibodies (daclizumab and basiliximab) increased from $0 \% 5$ years ago to $34 \%$ in 2002 . The use of either equine antithymocyte globulin or an anti-IL-2 receptor antibody appears to be the dominant strategy for induction in heart-lung transplantation.

\section{Trends in maintenance immunosuppression therapy prior to discharge in heart-lung transplantation}

As in heart transplantation, from 1993 to 2002 there have been no major changes in the general scheme of immunosuppression therapy prior to discharge for heart-lung transplant recipients. Shifts within the certain specific classes of immunosuppressants have not been as dramatic as seen with other organ types.

Calcineurin inhibitors. Calcineurin inhibitor use at the time of discharge remains essentially 100\%. Cyclosporine use has fallen from a high of $93 \%$ in 1994 to $62 \%$ in 2002. Of those who received cyclosporine, most received Neoral ${ }^{\circledR}$. A small proportion received either Sandimmune ${ }^{\circledR}$ 
or Gengraf ${ }^{\circledR}$. In contrast, the use of tacrolimus increased substantially. Tacrolimus is now used in $43 \%$ of heart-lung transplant recipients at the time of discharge.

Antimetabolites and rapamycin. The use of antimetabolites (azathioprine and mycophenolate mofetil) at the time of discharge has remained fairly constant over the past 10 years, ranging from $94 \%$ in 1993 to $95 \%$ in 2002 . Although the use of azathioprine has declined and the use of mycophenolate mofetil has proportionally risen, this has not occurred to the same extent as is seen in heart transplantation. In 2002, azathioprine was used in $52 \%$ of heart-lung transplant recipients at the time of discharge and mycophenolate mofetil was used in $48 \%$. The use of other agents such as rapamycin has not occurred in heart-lung transplant patients at the time of discharge.

Corticosteroids. The use of corticosteroids has fluctuated a little in the past decade (a low of $88 \%$ in 1996, a high of $100 \%$ in 1999-2002).

\section{Trends in maintenance immunosuppression therapy for the first year in heart-lung transplantation}

Calcineurin inhibitors. Cyclosporine or tacrolimus remain the cornerstones of maintenance immunosuppression in heart-lung transplant recipients. Over the past 10 years, there has been a trend towards less use of cyclosporine and more use of tacrolimus. Cyclosporine use has decreased from $81 \%$ in 1992 to $50 \%$ in 2001, with Neoral ${ }^{\circledR}$ being the most frequent formulation. During the same time interval, the use of tacrolimus has increased greatly, from $19 \%$ to $75 \%$.

Antimetabolites and rapamycin. Although antimetabolites are still used frequently within the first year after heart-lung transplantation, there has been a shift in practice over the last decade. The use of azathioprine declined from $97 \%$ in 1992 to $50 \%$ in 2001 . Concurrently, the use of mycophenolate mofetil rose to $60 \%$. The use of rapamycin increased to $10 \%$ in 2000 , however no first-year heart-lung patients received rapamycin in 2001.

Corticosteroids. The vast majority of heart-lung transplant recipients were treated with corticosteroids as part of maintenance therapy during the first year after transplantation. In 2001, 95\% of heart-lung transplant recipients received either prednisone or methylprednisolone.

In 2001, the most common maintenance therapy combination for the small numbers of heart-lung transplant recipients within the first year after transplantation consisted of corticosteroids, tacrolimus, and mycophenolate mofetil.

\section{Trends in antirejection treatment in heart-lung transplantation}

For first-year transplant recipients who require therapy for rejection, the use of corticosteroids has remained between
$80 \%$ and $100 \%$ in the small number of patients who have received heart-lung transplants. During the last 10 years, the use of therapy with antibodies varied from $10 \%$ to $20 \%$. When antibody therapy was used, muromonab-CD3, equine antithymocyte globulin, rabbit antithymocyte globulin and anti-IL-2 receptor antibodies were all used with no clear trends, given the small numbers of transplanted patients.

\section{Comparison of Organs}

\section{Trends in induction therapy}

The proportion of patients receiving induction therapy varied widely among organs (Figure 21). The highest use of induction therapy was reported for SPK and PAK transplants. Its use was reported in over $70 \%$ of the recipients in 2002 ; this was followed by PTA $(67 \%)$, kidney $(65 \%)$, intestine (57\%), and thoracic (over $40 \%$ ). Induction was not very common in liver transplants and was reported in only $18 \%$ of recipients in 2002 .

In the earlier years, muromonab-CD3 was the predominant induction agent used in most organs, except in thoracic transplantation, where the use of equine antithymocyte globulin was as common. A switch from equine antithymocyte globulin or muromonab-CD3 as the primary induction agent to IL-2 receptor antagonists (basiliximab and daclizumab) occurred in either 1998 or 1999 for kidney, kidney-pancreas, pancreas, liver, and intestine, followed by a switch from IL-2 receptor antagonists to rabbit antithymocyte globulin in 2000 or 2001. In 2002, rabbit antithymocyte globulin was used more often than IL-2 receptor antagonists for pancreas, kidney-pancreas, and intestine, whereas IL-2 receptor antagonists were used more commonly than rabbit antithymocyte globulin for liver and kidney transplants.

Both muromonab-CD3 and equine antithymocyte globulin were reported as common induction agents for thoracic organs in the earlier years and were replaced by IL-2 receptor antagonists in 2000 for lung and in 2001 for heart

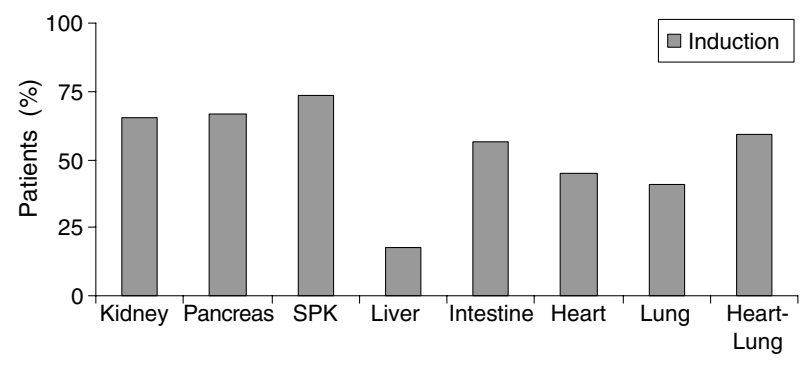

Source: 2003 OPTN/SRTR Annual Report, Table 5.6-13.6.

Figure 21: Comparative use of immunosuppression for induction prior to discharge, all organs, 2002. 
and heart-lung. While a switch to rabbit antithymocyte globulin occurred in 2002 for heart, IL-2 receptor antagonists remained the more common induction agent for lung and heart-lung in 2002.

\section{Trends in maintenance immunosuppression therapy prior to discharge}

Immunosuppressive regimens used for maintenance therapy at discharge varied by organ (Figures 22 and 23). The use of corticosteroids as discharge maintenance immunosuppression was reported in the majority of transplant recipients. Corticosteroids were used in over $87 \%$ of recipients of kidney, SPK, PAK, and thoracic transplants, and in over $70 \%$ of recipients of PTA. The use of corticosteroids in intestine transplants was reported in over $80 \%$ of recipients until 2002, where the use was reported in only $64 \%$ of recipients.

Cyclosporine was the most-used calcineurin inhibitor in most organs until the introduction of tacrolimus around 1994. A switch from cyclosporine to tacrolimus occurred in 1995 for pancreas, in 1996 for liver, in 1997 for SPK, and in 2001 for kidney. Cyclosporine remained the calcineurin inhibitor agent of choice for heart and heart-lung through 2002, whereas a switch to tacrolimus was noted for lung in 2002. For intestine, tacrolimus has been the predominant calcineurin inhibitor agent since 1994. In 2002, the proportion of recipients reported with tacrolimus as discharge maintenance immunosuppression was $63 \%$ for kidney, 95\% for PTA, 86\% for PAK, 87\% for SPK, 87\% for liver, $96 \%$ for intestine, 39\% for heart, $57 \%$ for lung, and $43 \%$ for heart-lung.

Azathioprine was the favored antimetabolite agent for most organs until the introduction of mycophenolate mofetil in late 1995. A switch from azathioprine to mycophenolate mofetil occurred in 1996 for kidney, pancreas, SPK, and intestine, in 1997 for liver, and in 1999 for heart. Azathioprine remained the antimetabolite agent of choice for lung and heart-lung transplants. In 2002, the propor-

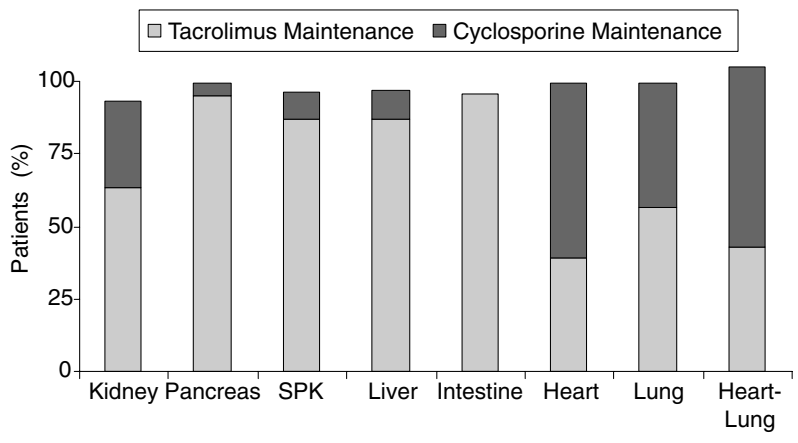

Source: 2003 OPTN/SRTR Annual Report, Table 5.6-13.6.

Figure 22: Comparative calcineurin inhibitor use for immunosuppression prior to discharge, all organs, 2002.

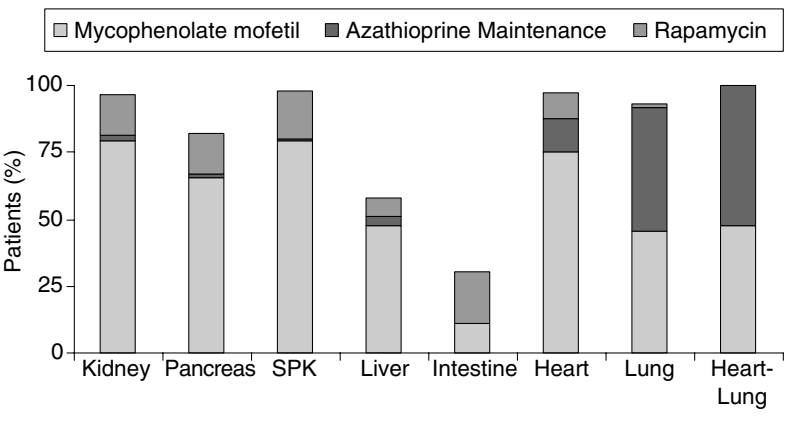

Source: 2003 OPTN/SRTR Annual Report, Table 5.6-13.6.

Figure 23: Comparative use of antimetabolites and rapamycin immunosuppression prior to discharge, all organs, 2002.

tion of recipients reported with mycophenolate mofetil as discharge maintenance immunosuppression was $79 \%$ for kidney, $64 \%$ for PTA, 74\% for PAK, 79\% for SPK, 48\% for liver, $11 \%$ for intestine, $75 \%$ for heart, $45 \%$ for lung, and $48 \%$ for heart-lung.

The use of rapamycin started to emerge in 2000 for all organs except heart-lung. In 2002, the use of rapamycin ranged from $15 \%$ to $20 \%$ in kidney, pancreas, SPK, and intestine transplants. During the same year, rapamycin was reported in $7 \%$ of liver, $10 \%$ of heart, and only $2 \%$ of lung transplant recipients.

\section{Trends in maintenance immunosuppression therapy between discharge and 1 year post-transplant}

The use of corticosteroids between discharge and 1 year after transplant was reported in the majority of transplant recipients. For transplants performed in 2001, the proportion of recipients with corticosteroids between discharge and 1 year ranged from $86 \%$ for intestine to $100 \%$ for lung.

Cyclosporine was the most reported maintenance calcineurin inhibitor between discharge and 1 year posttransplant in most organs until about 1994. A switch from cyclosporine to tacrolimus occurred for transplants performed in 1994 for PTA, in 1995 for liver, in 1996 for PAK, in 1997 for SPK, in 2000 for lung, and in 2001 for kidney and heart-lung. For heart, cyclosporine remained the calcineurin inhibitor agent of choice, whereas for intestine, tacrolimus has been the predominant calcineurin inhibitor agent since 1994. For transplants performed in 2001, the proportion of recipients reported with tacrolimus as maintenance immunosuppression between discharge and 1 year was 64\% for kidney, 99\% for PTA, 93\% for PAK, $89 \%$ for SPK and liver, 100\% for intestine, 43\% for heart, $69 \%$ for lung, and $75 \%$ for heart-lung.

A switch from azathioprine to mycophenolate mofetil occurred for transplants performed in 1995 for PTA, in 1996 for kidney, PAK, SPK, liver, and intestine, in 1997 for heart, 


\section{Dixon B. Kaufman et al.}

in 2001 for lung, and in 2000 for heart-lung. Although a switch from azathioprine to mycophenolate mofetil had occurred for intestinal transplants performed since 1996, azathioprine became the antimetabolite agent of choice again for transplants done in 2001. For lung, azathioprine remained the antimetabolite agent of choice, although it was used at a comparable rate to mycophenolate mofetil for transplants done in 2001 (50\% vs. 52\%). For transplants performed in 2001, the proportion of recipients reported with mycophenolate mofetil between discharge and 1 year was $80 \%$ for kidney, $75 \%$ for PTA, $79 \%$ for PAK, $78 \%$ for SPK, $50 \%$ for liver, $81 \%$ for heart, $52 \%$ for lung, $60 \%$ for heart-lung, and only $3 \%$ for intestine.

For most organs, the use of rapamycin between discharge and 1 year post-transplant started to emerge for transplants done in 1999 or 2000. For transplants performed in 2001, the use of rapamycin between discharge and 1 year ranged from 0\% for heart-lung to $29 \%$ for PAK transplants.

\section{Trends in antirejection treatment between discharge and 1 year post-transplant}

Although the proportion of recipients reported with antibody treatment for rejection may have fluctuated over the years, overall there was a decreasing trend in its use between transplant and 1 year post-transplant for most organs. For transplants done in 1994, for example, 53\% of kidney, over $60 \%$ of SPK and pancreas, about $30 \%$ of liver and intestine, and over $17 \%$ of thoracic transplants were reported with nonsteroid antirejection therapy between discharge and 1 year post-transplant. In contrast, in $2002,37 \%$ of kidney, $62 \%$ of PTA, $63 \%$ of PAK, $30 \%$ of SPK, $17 \%$ of liver, $16 \%$ of heart, $12 \%$ of lung, and $0 \%$ of heart-lung transplants were reported with noncorticosteroid antirejection therapy between discharge and 1 year post-transplant. Interestingly, the proportion of intestinal transplants treated for rejection remained at 35\%.

The use of corticosteroids for antirejection therapy was more common than use of an antibody agent for most organs. Corticosteroids were the most common treatment for rejection between discharge and 1 year post-transplant for transplants done in 2001 for at least $80 \%$ of kidney, PAK, SPK, liver, and intestine recipients. Over $90 \%$ of PTA, heart, lung, and heart-lung transplants in 2001 were reported to have been performed with corticosteroids.

For most organs, muromonab-CD3 was the most common antibody therapy used to treat rejection between discharge and 1 year post-transplant during the earlier years. For transplants done in 2001, the proportion of recipients reported with muromonab-CD3 was 11\% for kidney, $29 \%$ for PTA, $28 \%$ for PAK, $12 \%$ for SPK, 33\% for intestine, and less than $10 \%$ for liver, heart, and lung transplants. ALG was also used in most organs except liver and intestine until about 1994, when it was replaced by equine antithymocyte globulin. The use of equine antithymocyte globulin as an antirejection agent was more common in thoracic transplants during the more recent years and was comparable with the use of muromonab-CD3.

The use of rabbit antithymocyte globulin as an antirejection therapy started to be reported for transplants performed in 1999 for most organs. For transplants performed in 2001, the proportion of recipients reported with rabbit antithymocyte globulin rejection therapy between discharge and 1 year post-transplant was $18 \%$ for kidney, $17 \%$ for SPK, $38 \%$ for PTA, 35\% for PAK, $5 \%$ for liver and heart, $8 \%$ for intestine, and $6 \%$ for lung.

\section{Summary}

This review has documented marked changes in the use of immunosuppressive agents in solid organ transplantation over the past decade. Several consistencies have emerged with respect to the use of maintenance immunosuppression. There has been a transition within the calcineurin inhibitor era from the dominant use of cyclosporine to tacrolimus and the near ubiquitous use of mycophenolate mofetil over azathioprine. Chronic use of corticosteroids remains a mainstream practice despite increased attention to successful steroid-sparing strategies. Induction therapy is being incorporated into immunosuppression protocols with increasing frequency. The agents employed are mostly IL-2 receptor antagonists, but clear trends indicate that T-cell depletion is being used more often. The result of these changes in clinical practice seems to indicate that the short-term outcomes have improved based on the observation that rates of rejection within the first year post-transplant have diminished (20). Future surveys of trends in immunosuppression use are unlikely to show a great deal of change over the next few years, but subtle signs of immunosuppression minimization (diminished use of steroids) and new induction therapies (e.g. Campath ${ }^{\circledR}$ ) are likely to surface. Ultimately, one would like to know how today's choices of immunosuppression will affect outcomes in the long term. Future reports will be able to answer those questions.

\section{References}

1. Dickinson DM, Bryant PC, Williams MC et al. Transplant data: sources, collection, and caveats. Am J Transplant 2004; 4(Suppl. 9): 13-26.

2. Wolfe RA. Schaubel DE, Webb RL et al. Analytical approaches for transplant research. Am J Transplant 2004; 4(Suppl. 9): 106-113.

3. Pirsch JD, Miller J, Deierhoi MH, Vincenti F, Filo RS. A comparison of tacrolimus (FK506) and cyclosporine for immunosuppression after cadaveric renal transplantation. FK506 Kidney Transplant Study Group. Transplantation 1997; 63: 977-983.

4. Johnson C, Ahsan N, Gonwa T et al. Randomized trial of tacrolimus (Prograf) in combination with azathioprine or mycophenolate mofetil versus cyclosporine (Neoral) with mycophenolate mofetil after cadaveric kidney transplantation. Transplantation 2000; 69: 834-841. 
5. Shapiro R, Jordan ML, Scantlebury VP et al. A prospective, randomized trial of tacrolimus/prednisone versus tacrolimus/prednisone/mycophenolate mofetil in renal transplant recipients. Transplantation 1999; 67 (3): 411-415.

6. Margreiter R. European Tacrolimus vs Ciclosporin Microemulsion Renal Transplantation Study Group. Efficacy and safety of tacrolimus compared with ciclosporin microemulsion in renal transplantation: a randomised multicentre study. Lancet 2002; 3359: 741-746.

7. Matas AJ, Rancharan T, Paraskevas S et al. Rapid discontinuation of steroids in living donor kidney transplantation: a pilot study. Am J Transplant 2001; 1: 278.

8. Cole E, Landsberg D, Russell D et al. A pilot study of steroid-free immunosuppression in the prevention of acute rejection in renal allograft recipients. Transplantation 2001; 72: 845.

9. Vincenti F, Monaco A, Grinyo J et al. A multicenter randomized prospective trial of steroid withdrawal in renal transplant recipients receiving basiliximab, cyclosporine microemulsion and mycophenolate mofetil. Am J Transplant 2003; 3: 306-311.

10. Gruessner AC, Sutherland DER. Pancreas transplant outcomes for United States (US) and non-US cases as reported to the United Network for Organ Sharing (UNOS) and the International Pancreas Transplant Registry (IPTR) as of October, 2002. In:Cecka JM, Terasaki PI (eds). Clinical Transplants 2002, Chapter 4. Los Angeles: UCLA Tissue Typing Laboratory 2004: pp. 41-77.

11. Kaufman DB. Induction Therapy, in Transplantation of the Pancreas,Gruessner RWG, Sutherland DER (eds). New York: Springer-Verlag, 2003.

12. Kaufman DB, Burke GW, Bruce DS et al. A prospective, randomized, multi-center trial of antibody induction therapy in simultaneous pancreas-kidney transplantation. Am J Transplant 2003; 3: 855-864.
13. Kaufman DB, Leventhal JR, Koffron AJ et al. A prospective study of rapid corticosteroid elimination in simultaneous pancreas-kidney transplantation: Comparison of two maintenance immunosuppression protocols: tacrolimus/mycophenolate mofetil versus tacrolimus. Transplantation 2002; 73: 169177.

14. Jordan ML, Chakrabarti P, Luke P et al. Results of pancreas transplantation after steroid withdrawal under tacrolimus immunosuppression. Transplantation 2000; 69: 265.

15. Gruessner RW, Sutherland DE, Parr E, Humar A, Gruessner AC. A prospective, randomized, open-label study of steroid withdrawal in pancreas transplantation-a preliminary report with 6-month follow-up. Transplant Proc 2001; 33: 1663-1664.

16. Trotter JF, Wachs M, Bak $T$ et al. Liver transplantation using sirolimus and minimal corticosteroids (3-day taper). Liver Transpl 2001; 4: 343-351.

17. Ringe B, Braun F, Schutz E et al. A novel management strategy of steroid-free immunosuppression after liver transplantation: efficacy and safety of tacrolimus and mycophenolate mofetil. Transplantation 2001; 71: 508-515

18. Wiesner RH. A long-term comparison of tacrolimus (FK506) versus cyclosporine in liver transplantation. United States FK506 Study Group. Transplantation 1998; 66 (4): 493499.

19. King-Biggs MB, Dunitz JM, Park SJ, Kay Savik S, Hertz MI. Airway anastomotic dehiscence associated with use of sirolimus immediately after lung transplantation. Transplantation 2003; 75: $1437-1443$.

20. Cherikh WS, Kauffman HM, Maghirang J, Bleyer AJ, Johnson CP. A comparison of discharge immunosuppressive drug regimens in primary cadaveric kidney transplantation. Transplantation 2003: 76: 463-470. 